cumulative over the course of development. This further suggests that the presentation of psychosis represents a culmination of an ongoing interaction between an individual and his/her environment. This remains the only reasonable explanation for the variation in incidence rates, particularly those reported for migrant populations in Britain and Europe (Hutchinson \& Haasen, 2004). Interactions between perceptions of self, cognitive processes and the features of a modern urban environment underlie social development. The relative weighting of vulnerability and resilience factors is a function of this interaction and must in turn be affected by wider social issues such as racism, socioeconomic opportunity and perceived social isolation. There is also the generational transfer of unfulfilled expectations and distrust of institutional structures. The problems in mental health for migrants in Britain are mirrored in the education and criminal justice systems (Modood et al, 1997). This suggests a developmental trajectory that is affected by social and generational realities and at the same time increases the risk of presentation with psychotic symptoms.

This would mean that the risk exposure for psychosis lies not specifically in the urban environment but in the way this environment generates and/or facilitates a life course that ultimately disadvantages those whose vulnerability is not compensated for by the support of their social environment. This is also influenced by the individual's perception of the negative experiences of the ethnic and socio-cultural groups with which they identify in both the narrow family and community sense as well as the wider national and international sense.

There might therefore be a need to reconstruct the neurodevelopmental model which has led to a preoccupation with the biology of psychosis to include a social developmental model that can demonstrate how the neurobiological endpoint of psychosis can have both biological and social origins.

Hutchinson, G. \& Haasen, C. (2004) Migration and schizophrenia. The challenges for European psychiatry and implications for the future. Social Psychiatry and Psychiatric Epidemiology, 39, 350-357.

Modood, T., Berthoud, R., Lakey, J., et al (1997) Ethnic Minorities in Britain: Diversity and Disadvantage. London: Policy Studies Institute.
Tsuang, M.T., Stone, W. S. \& Faraone, S.V. (200I) Genes, environment and schizophrenia. British journal of Psychiatry, 178 (suppl. 40), sl8-s24.

Van Os, J. (2004) Does the urban environment cause psychosis? British Journal of Psychiatry, 184, 287-288.

G. Hutchinson Psychiatry Unit, Department of Clinical Medical Sciences, University of the West Indies, Champs Fleurs, Trinidad

C. Morgan Division of Psychological Medicine, Institute of Psychiatry, De Crespigny Park, London SE5 8AF, UK

Van Os (2004) discusses the implication from the epidemiological research by Sundquist et al (2004) that psychosis may indeed be due to urban toxicity. The dose-response increase in urbanicity with schizophrenia does incline to an explanation of causation rather than association. The discussion of a set of environmental factors acting between birth and the onset of psychosis (child and adolescence) should have led to a discussion of the role that cannabis plays in the early onset of psychosis. This link between substance use and urbanicity was, however, not discussed in the editorial.

The clue to an ecological exposure lies in the early use of cannabis. Arseneault et al (2002) in a prospective study found an association between early use of cannabis (by the age of 15) and an increased risk of psychosis for 1037 children born in New Zealand. This aetiological factor interacts with the increased social fragmentation, social inequality and social isolation found with greater urbanicity. The cognitive vulnerabilities for psychosis have a strong social environmental aetiology, and a link needs to be made between models of urban toxicity and increased early cannabis use.

Arseneault, L., Cannon, M., Poulton, R., et al (2002) Cannabis use in adolescence and risk for adult psychosis: longitudinal prospective study. BMJ, 325, 1212-1213.

Sundquist, K., Frank, G. \& Sundquist, J. (2004)

Urbanisation and incidence of psychosis and depression. Follow-up study of 4.4 million women and men in Sweden. British Journal of Psychiatry, 184, 293-298.

Van Os, J. (2004) Does the urban environment cause psychosis? British Journal of Psychiatry, I84, 287-288.

K. Marlowe Early Psychosis Team, Counties Manukan DHB, South Auckland, New Zealand. E-mail: Karl.Marlowe@middlemore.co.nz

\section{Memantine as a neuroprotective treatment in schizophrenia}

Phospholipid metabolism occurs in cell (including neuron) membranes and although regional differences are described by Jensen et al (2004), these are not neurotransmitter-specific. This research suggests increased phospholipid metabolism in the anterior cingulate area of people with schizophrenia.

Jensen et al suggest that this is supportive evidence for a neurodegenerative mechanism in schizophrenia. They also review the effects of neuroleptic and anxiolytic (including benzodiazepine) medications on brain phosphorus metabolism.

Memantine is a drug currently licensed for use in people with moderate to severe Alzheimer's dementia. It is a non-competitive, low-affinity $N$-methyl-Daspartate (NMDA) antagonist. (The NMDA receptor is a class of glutamate receptor.) Glutamate-mediated excitotoxicity and/or receptor dysfunction is involved in the pathogenesis of several neuropsychiatric and neurological disorders. Memantine partially blocks these NMDA receptors, preventing a neurotoxic influx of calcium. Theoretically, it is neuroprotective for glutamate-receiving neurons.

Given its mode of action, it should theoretically be more effective in the early stages of neurodegenerative disorders such as Alzheimer's dementia. On these theoretical grounds it may also be neuroprotective for people with schizophrenia.

Jensen, J. E., Miller, J., Williamson, P. C., et al (2004) Focal changes in brain energy and phospholipid metabolism in first-episode schizophrenia: ${ }^{31} \mathrm{P}-\mathrm{MRS}$ chemical shift imaging study at 4 Tesla. British Journal of Psychiatry, 184, 409-4I5.

G. S. J. Rands Camden and Islington Mental Health and Social Care Trust, and Department of Mental Health Sciences, Royal Free and UCL Medical School, Archway Campus, Highgate Hill, London NI9 5NF, UK.

E-mail: Gianetta.rands@candi.nhs.uk

Authors' reply: Memantine, as described by Dr Rands, would appear to be a suitable candidate as a neuroprotective agent for people with schizophrenia, based on its NMDA-receptor-blocking properties. This drug is currently in use as a treatment for people with moderate to severe Alzheimer's dementia.

As shown by Theberge et al (2002, 2003), glutamate levels in first-episode schizophrenia are higher than normal in the anterior cingulate and lower than normal in this same region in the chronic stages of illness. As shown in this same work, $\mathrm{N}$ acetylaspartate levels correlate negatively 